\title{
RDA - Das Regelwerk für die Zukunft
}

Nach langer Vorbereitung und vielen Diskussionen im Vorfeld wurde zum 1. Oktober 2015 das Regelwerk des 21. Jahrhunderts „Resource Description and Access" (RDA) in der deutschsprachigen Bibliothekswelt eingeführt und löste damit das bisherige Standardregelwerk "Regeln für die alphabetische Katalogisierung" (RAK) ab. Anders als RAK ist RDA nicht mehr auf Zettelkataloge ausgerichtet, sondern ausschließlich auf elektronische Kataloge.

\section{Entwicklung und Einführung}

Bereits im Jahr 2004 begannen die Arbeiten an einem Nachfolgeregelwerk für AACR2, das Standardwerk für den angloamerikanischen Raum. AACR3 blieb aber eine weitere Ausgabe der AngloAmerican Cataloguing Rules (AACR). Schon 2001 hatte der Standardisierungsausschuss der Deutschen Nationalbibliothek beschlossen, auf internationale Regelwerke und Formate umzusteigen. 2005 kam es zu einer Neuausrichtung und einem an aktuelle Bedürfnisse angepassten Regelwerk unter neuem Titel "Resource Description and Access (RDA)", was übersetzt werden könnte mit "Ressourcen beschreiben und zugänglich machen". Eine offizielle deutsche Übersetzung gibt es nicht.

Bestimmte Anforderungen wurden an RDA gestellt. Das Regelwerk sollte

- international ausgerichtet und webfähig sein

- in Bibliotheken, Archiven und Museen Anwendung finden

- für alle Arten von Ressourcen und Inhalten gelten

- formatunabhängig formuliert sein

Die Art der bibliografischen Beschreibung nach RDA soll dem Bibliotheksbenutzer ermöglichen, die gewünschte Ressource aufgrund seiner Suchkriterien

- zu finden

- zu identifizieren

- auszuwählen

- und Zugang zu erhalten
2010 erschien die erste Ausgabe des Regelwerks RDA. Seit 2013 katalogisieren Nationalbibliotheken und weitere große Bibliotheken (z.B. Library of Congress, British Library, National Library of Australia) nach RDA.

Der Standardisierungsausschuss der Deutschen Nationalbibliothek hat sich 2011 für die Einführung der RDA im deutschsprachigen Raum ausgesprochen und den Umstieg beschlossen. Die vom Standardisierungsausschuss eingesetzte Arbeitsgruppe RDA (AG RDA), bestehend aus Vertretern der Arbeitsgemeinschaft der Verbundsysteme und der Deutschen Nationalbibliothek, war für die Vorbereitung und Implementierung der RDA, für die Erarbeitung der Anwendungsrichtlinien für die Praxis in Deutschland, Österreich und der Schweiz (D-A-CH) sowie für die Ausarbeitung der Schulungsunterlagen zuständig.

Die Einführung der RDA geschah in zwei Stufen: Im Herbst 2014 wurde RDA für die Erfassung von Normdaten eingeführt, im Herbst 2015 folgte der Umstieg bei den Titeldaten.

\section{Schulungskonzept des SWB}

Das Konzept zu den Schulungen im deutschsprachigen Raum wurde von allen Partnern der AG RDA gemeinsam erarbeitet. Es gliedert sich in sechs Module

- Modul 1: Grundlagen der RDA

- Modul 2: Basiswissen Katalogisierung (Theorie)

- Modul 3: Basiswissen Katalogisierung:

Monografien und fortlaufende Ressourcen (Praxis)

- Modul 4: Normdaten

- Modul 5A: Aufbauwissen Katalogisierung: Monografien

- Modul 5B: Aufbauwissen Katalogisierung: Fortlaufende Ressourcen, ZDB

- Modul 6: Spezialschulungen

Die AG RDA arbeitete zu diesen Modulen formatneutrale und systemunabhängige Schulungsmaterialien aus und stellte diese in Form von Power-PointPräsentationen und PDF-Skripten in das RDA-Informations-Wiki der Deutschen Nationalbibliothek. 
Das Bibliotheksservice-Zentrum Baden-Württemberg (BSZ) arbeitete für den Südwestdeutschen Bibliotheksverbund (SWB) einen Zeitplan und ein eigenes Schulungskonzept aus, da alle im SWB katalogisierenden Mitarbeiterinnen und Mitarbeiter RDA-Schulungen benötigten. Bereits Ende 2014 wurde eine Anwendergruppe (AG Schulung) gebildet, bestehend aus Vertretern der Universitätsund Landesbibliotheken und Mitarbeitern des BSZ. Aus der Württembergischen Landesbibliothek wurden drei Kolleginnen aus der Medienbearbeitung entsandt.

Zur Vorbereitung wurde die E-Learning-Plattform Ilias eingerichtet, in der die formatbezogenen Schulungsunterlagen zum Selbststudium zur Verfügung gestellt wurden. Anhand von eingescannten Titelblättern und Aufgaben konnten die RDAKenntnisse umgesetzt und praktisch in der RDASchulungsdatenbank des SWB erfasst werden. Die Plattform diente darüber hinaus als Diskussionsforum, als Austausch und für Korrekturwünsche. Drei Präsenzsitzungen der AG Schulung wurden einberufen.

Die Mitglieder der AG Schulung hatten die Aufgabe, die Schulungen in ihren Bibliotheken zu organisieren, weitere Multiplikatoren auszubilden und als Ansprechpartnerinnen und Ansprechpartner innerhalb der Bibliotheken und des BSZ den RDA-Umstieg 2015 zu begleiten.

Das erste eintägige Meeting fand am 3. Februar 2015 in der Universitätsbibliothek Stuttgart statt. Es befasste sich u.a. mit den Themen Schulungskonzept, Zeitplan, E-Learning-Angebote, Einführung in die RDA und Benutzung des RDA-Toolkits.

Vom 11. bis 13. Mai fand das zweite Treffen der AG Schulung in Stuttgart im Schulungsraum des BSZ statt. Schulungsinhalte waren Modul 2 und 3 mit Übungsbeispielen.

Die dritte AG Schulung wurde vom 8. bis 11. September ebenfalls im Schulungsraum des BSZ veranstaltet. In dieser Schulung wurden die Module 4 und $5 \mathrm{~A}$ vertieft.

Vor den jeweiligen Sitzungen wurden die formatbezogenen PDF-Skripte und Power-Point-Präsen- tationen auf der E-Learning-Plattform Ilias zum Selbststudium veröffentlicht.

\section{Schulungskonzept der WLB}

Parallel zu den Präsenzsitzungen der AG Schulung liefen die Vorbereitung, Planung und Durchführung der RDA-Schulungen in der WLB. Drei weitere Kolleginnen aus der Medienbearbeitung konnten von Anfang an zur Mitarbeit als Multiplikatoren gewonnen werden. Das RDA-Team traf sich anfangs wöchentlich, später je nach Bedarf auch öfter zur Vertiefung der RDA-Kenntnisse, zur Besprechung der Schulungsunterlagen und zum Üben in der RDA-Schulungsdatenbank. Die Sitzungen der AG Schulung konnten im Team vorbereitet und die Informationen und Kenntnisse aus den Schulungen wiederum an alle Multiplikatoren weitergegeben werden.

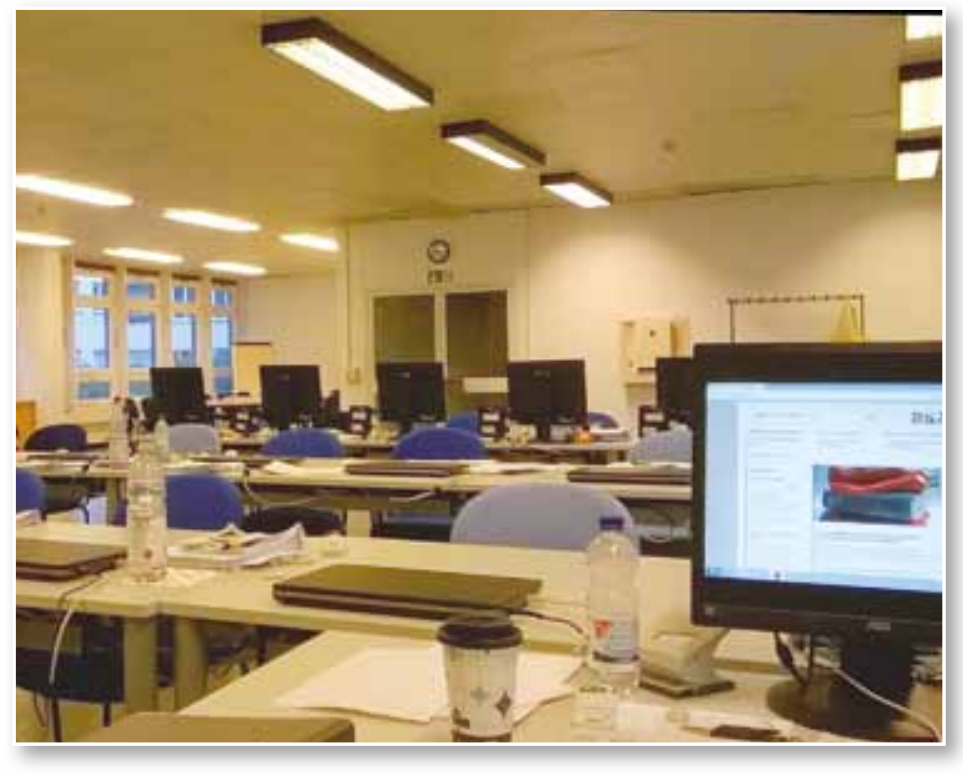

Abb. 1: Schulungsraum des Bibliotheksservice-Zentrums in Stuttgart

Die Schulungen für 56 Kolleginnen und Kollegen der WLB und 16 Kolleginnen und Kollegen aus sechs kleineren Bibliotheken, die nach Rücksprache vom BSZ zugeteilt wurden, mussten frühzeitig organisiert werden.

Angesetzt wurden 2 Schulungsblöcke für die Module 1-3 und 4-5A. Für die Anmeldung zu den beiden Schulungsblöcken wurde ein Anmeldeformular im Intranet verbreitet. Während noch die dritte Präsenzsitzung der AG Schulung im September 2015 stattfand, liefen in der WLB auch schon die ersten Schulungen zu Modul 1-3. Aus dem Team der sechs Multiplikatoren waren jeweils 
zwei Kolleginnen verantwortlich für einen der drei Schulungsblöcke: Modul 1-3, Modul 4-5A und Modul 5B. Sie konnten sich somit intensiv auf die jeweiligen internen Schulungen vorbereiten.

Die ganztägigen Schulungen für die Module 1-3 fanden in den Kalenderwochen 36-40 von Montag bis Donnerstag statt. Die Module beinhalteten folgende Themen

- Modul 1: Grundlagenmodelle der RDA (FRBR), Regelwerk und Terminologie

- Modul 2: Theoretisches Basiswissen für die Katalogisierung: Primäre Informationsquellen für die Katalogisierung nach RDA; Standard-Elemente-Set; RDA-Elemente Inhaltstyp, Medientyp, Datenträgertyp; Festlegungen zu Sprache und Schrift; Abgrenzung von Publikationstypen (Monographien, fortlaufende und integrierende Ressourcen)

- Modul 3: Basiswissen Katalogisierung (Praxis): Katalogisieren von einfachen Aufnahmen unterschiedlicher Materialarten; Anwendung der RDA-Regeln und der Anwendungsrichtlinien für den deutschsprachigen Raum (D-A-CH) auf allen Ebenen der zusammengesetzten Beschreibung; Nutzung von Normdaten

Parallel dazu bereiteten sich 2 weitere Multiplikatoren, die auch an der dritten Präsenzsitzung der AG Schulung im September teilgenommen hatten, auf den zweiten Schulungsblock für Modul 4 und 5A in den Kalenderwochen 42-46 vor. Inhalte der vier ganztägigen Schulungstage waren

- Modul 4: Normdaten: Ergänzungen zu Körperschaften und Konferenzen; Normdatensätze für Werke

- Modul 5A: Aufbauwissen Katalogisierung (Monographien): Anwendung der RDA und der Anwendungsrichtlinien D-A-CH auf mehrteilige Monographien, Zusammenstellungen in umfassender und analytisch-hierarchischer Beschreibung, Begleitmaterial, Integrierende Ressourcen, Reproduktionen, Bildbände, Konferenzen, Körperschaften als geistige Schöpfer, Werk-zu-Werk-Beziehungen

Als BWZ-Zentrale hatte die WLB die Aufgabe, Modul 5B: Aufbauwissen Katalogisierung (fortlau- fende Ressourcen) zu vermitteln. Die Schulungen fanden ganztags in den Kalenderwochen 48-50 in Stuttgart statt. 33 Teilnehmer aus baden-württembergischen Bibliotheken hatten sich für jeweils fünf Schulungstage angemeldet. Vorausgesetzt wurden die Kenntnisse aus Modul 1-3.

Die beiden Kolleginnen aus dem Zeitschriftenbereich hatten im Juni 2015 an einer einwöchigen Multiplikatoren-Schulung der Zeitschriftendatenbank (ZDB) in Berlin teilgenommen. Eine der beiden Kolleginnen nahm auch an den drei Präsenzsitzungen der AG Schulung teil.

Wegen der beengten räumlichen Situation in der WLB, bedingt durch die Abrissmaßnahmen für den Erweiterungsbau, wurde uns dankenswerterweise der Schulungsraum des BSZ für mehrere Wochen zur Verfügung gestellt.

\section{Regelwerk und RDA-Toolkit}

Anders als frühere Regelwerke steht RDA nur noch online im Online-Tool „RDA-Toolkit” zur Verfügung. Eine gedruckte deutschsprachige Ausgabe gibt es nicht. Im RDA-Toolkit ist u.a. die aktuelle deutsche Version des Regelwerks enthalten und die von der AG RDA erarbeiteten Anwendungsrichtlinien für den deutschsprachigen Raum (D-A-CH). Diese sind über den Button D-A-CH an der entsprechenden Regelwerksstelle verlinkt. Die Anwendungsrichtlinien (D-A-CH) mit Anwendungsregeln, Erläuterungen und Beispielen sind in der Praxis zu berücksichtigen.

Der Regelwerkstext ist nicht statisch, Aktualisierungen sind vorgesehen. Im

Februar-Release 2016 wurden Änderungen und Ergänzungen bei den Anwendungsrichtlinien (D-A$\mathrm{CH}$ ) eingearbeitet und veröffentlicht.

Mittlerweile ist das Regelwerk in mehrere Sprachen übersetzt worden.

RDA regelt die bibliografische Beschreibung aller Materialarten, unabhängig vom Datenträger. Es gibt keine Sonderregelwerke wie zu RAK-Zeiten. Zugriff auf das RDA-Toolkit haben die Bibliotheken und Informationseinrichtungen in Deutschland, Österreich und der Schweiz über die Lizenz des D-A-CH-Konsortiums. 


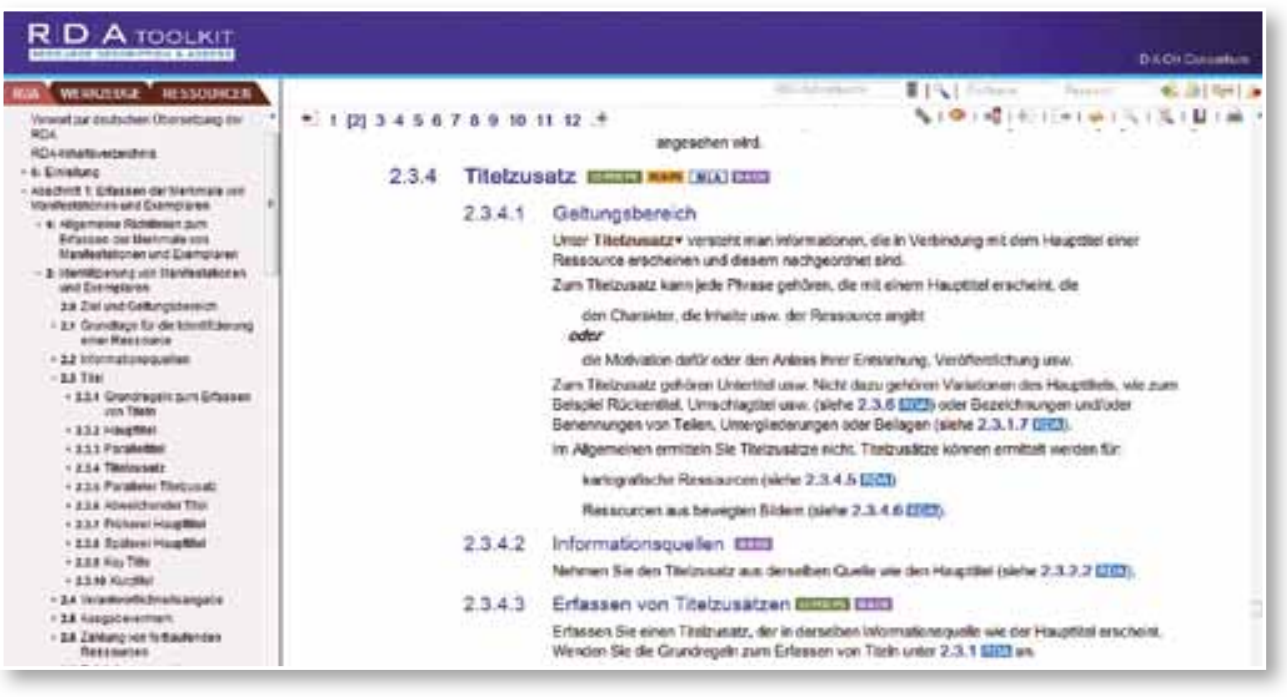

Abb. 2: Screenshot aus dem RDA-Toolkit (www.rdatoolkit.org), verwendet mit Genehmigung der RDA-Verleger (American Library Association, Canadian Library Association, und CILIP: Chartered Institute of Library and Information Professionals)

RDA beruht auf dem theoretischen Datenmodell FRBR (Functional Requirements for Bibliographic Records). Dieses spiegelt sich im Aufbau wider. Der Regelwerkstext ist klar strukturiert. Eine Einführung gibt es in Kapitel 0. Die beiden Hauptteile sind in Abschnitte gegliedert, wobei sich die Abschnitte 1 bis 4 mit den Merkmalen der Entitäten (Manifestation und Exemplar, Werk und Expression, Person, Familie und Körperschaft), die Abschnitte 5 bis 10 mit den Beziehungen zwischen den Entitäten beschäftigen. Wichtig sind die Anhänge mit Richtlinien, z.B. für Groß- und Kleinschreibung der englischen und sonstigen Sprachen, Abkürzungen, Adelstitel und Beziehungskennzeichen.

Ungewohnte neue Begriffe - Entitäten, Merkmale, Beziehungen sowie Werk, Expression, Manifestation und Exemplar - ziehen sich durch das Regelwerk. Aber es gibt noch viele andere Fachausdrücke, die mit RDA eingeführt wurden und an die man sich gewöhnen muss. Dazu gehören, z.B. Kern- und Zusatzelemente, Sucheinstiege, zusammengesetzte Beschreibung, umfassende Beschreibung, analytische Beschreibung, hierarchische Beschreibung, geistiger Schöpfer, Inhaltstyp, Medientyp, Datenträgertyp, Beziehungskennzeichen. Einige Termini wurden umbenannt, z.B. Verantwortlichkeitsangabe anstatt Verfasserangabe, Veröffentlichungsangabe anstatt Erscheinungsvermerk, Haupttitel anstatt Hauptsachtitel, Monografische Reihe anstatt Schriftenreihe, Anmerkungen anstatt Fußnoten. Früheres RAK-Vokabular, z.B.
Einheitssachtitel, allgemeine Materialbenennung, Ansetzungssachtitel, begrenztes Sammelwerk, Urheber gibt es in RDA nicht mehr.

Titelaufnahmen werden im SWB nach RDA-Standard als zusammengesetzte Beschreibung erstellt. Das bedeutet, in einem gemeinsamen Titeldatensatz sind alle Merkmale der Ebenen Werk, Expression und Manifestation erfasst. Wie bisher auch werden in diesem gemeinsamen Datensatz Beziehungen zu Personen und Körperschaften verlinkt.

Ein wichtiger Aspekt in RDA ist das „Erfassen“ und "Übertragen“. Beim Übertragen werden bestimmte Elemente der vorliegenden Manifestation vorlagegetreu abgeschrieben unter Beachtung der Regelungen für Sprache und Schrift, Groß- und Kleinschreibung, Zahlen, Abkürzungen, Initialen, Zeichensetzung, diakritische Zeichen, Symbole, Schreib- und Druckfehler. Dazu gehören Titel und Titelzusätze, Verantwortlichkeitsangaben, Ausgabevermerk, Erscheinungsorte und Verlagsnamen. Durch diese Art der Beschreibung kann die Ressource wiedererkannt und identifiziert werden.

\section{Erste praktische Erfahrungen}

Am 5. Oktober 2015 begann das RDA-Zeitalter im SWB. Wer bereits geschult war, konnte die Kenntnisse praktisch umsetzen. Zuvor mussten allerdings in der Katalogisierungsdatenbank des SWB die technischen Voraussetzungen zur Implementierung geschaffen werden. Für eine Übergangszeit können in Ausnahmefällen weiterhin RAK-Aufnahmen erstellt werden. Dieses RAK-Interim gilt für Neuaufnahmen juristischer, religiöser, kartographischer Werke und Alter Drucke bis RDA-Regeln vorliegen. Spezialschulungen und formatbezogene Schulungsunterlagen dafür sind 2016 geplant. Die Spezialschulung für Werke der Musik fand Ende Januar 2016 statt. 


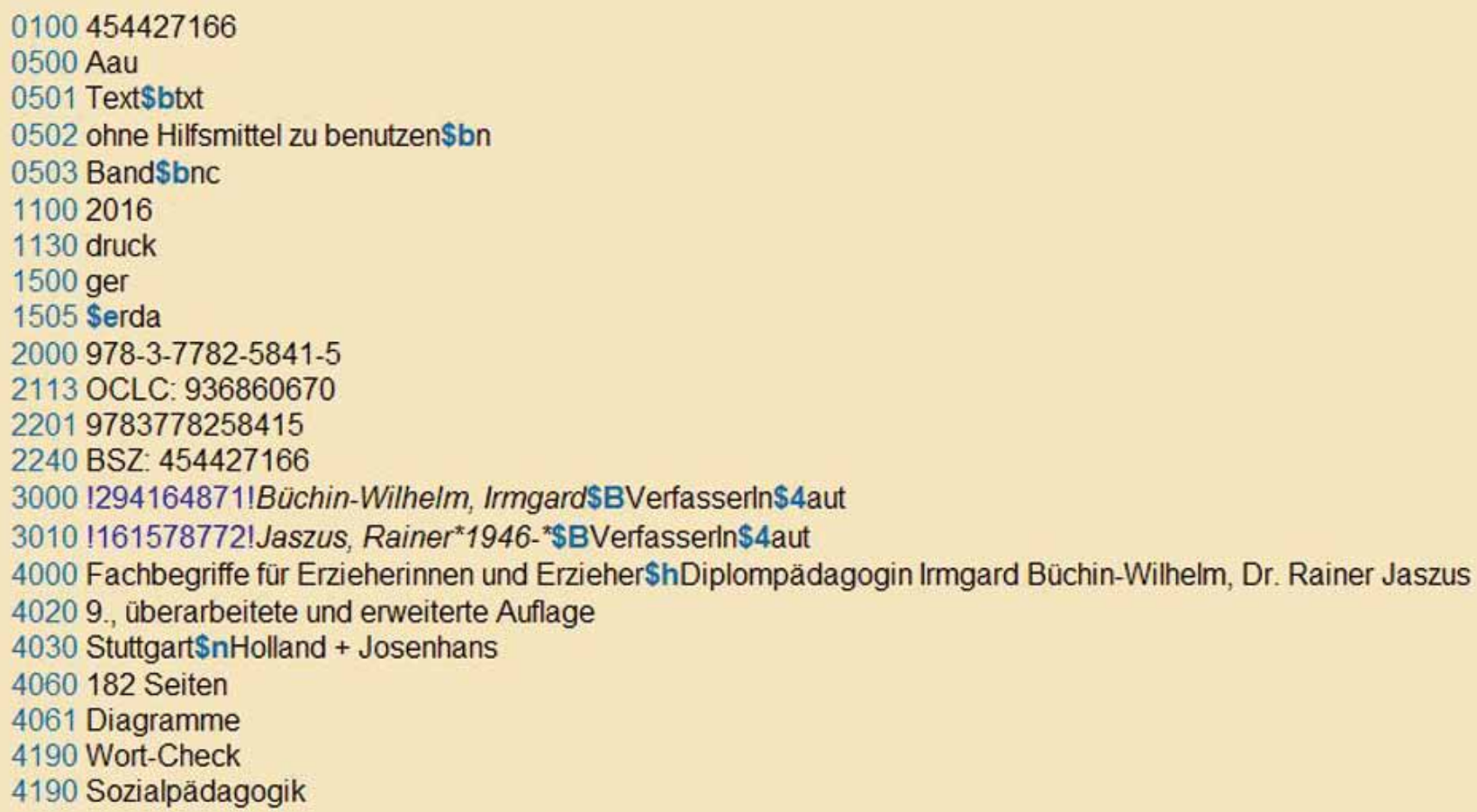

Abb. 3: SWB-Titelaufnahme nach RDA

Die llias-Plattform des SWB kann über die Regelwerkseinführung hinaus genutzt werden und bietet neben den Schulungsunterlagen wichtige Informationen zur praktischen Anwendung im SWB und zu wichtigen Änderungen im Regelwerkstext und in D-A-CH. Sie enthält auch Arbeitshilfen zu spezifischen Themen und dient zusätzlich als Kommunikationsforum bei Fragen oder Bemerkungen bezüglich RDA.

Verständlich geschrieben sind die Blog-Beiträge von Frau Professor Heidrun Wiesenmüller "Drei Minuten RDA", die Katalogisierungsprobleme thematisch betrachten. Das Lehrbuch „Basiswissen RDA, eine Einführung für deutschsprachige Anwender" wurde von Heidrun Wiesenmüller und Silke Horny gemeinsam verfasst.

Bei der praktischen Anwendung des RDA-Standards kommen viele Fragen und Unsicherheiten auf, die es zu klären gilt. In einigen Fällen muss das BSZ zu Rate gezogen werden. Auch seitens der Katalog-AG und der AG RDA besteht noch Handlungsbedarf bei Katalogisierungsfragen nach RDA.

RDA ist angelegt nach dem Prinzip "Cataloguer's Judgement" und bietet dem Katalogisierer mehr Entscheidungsmöglichkeiten und Freiheiten als
RAK. Die Anwendungsrichtlinien für den deutschsprachigen Raum (D-A-CH) legen fest, ob eine Alternative oder Option im Regelwerkstext angewendet werden soll oder nicht. Teilweise wird das Anwenden aber ins Ermessen des Katalogisierers gestellt.

Zu wünschen ist, dass die Neuerungen, die RDA dem Katalogisierer und Bibliotheksbenutzer bietet, im elektronischen Zeitalter maschinell in den Online-Katalogen umgesetzt werden.

\section{Brigitte Herrmann}

Verwendete Quellen:

- RDA-Toolkit http://access.rdatoolkit.org/

- RDA-Informations-Wiki der Deutschen Nationalbibliothek https://wiki.dnb.de/display/RDAINFO/ RDA-Info

- Heidrun Wiesenmüller und Silke Horny: Basiswissen RDA, eine Einführung für deutschsprachige Anwender. Berlin 2015 (gedruckt und als E-Book)

- Heidrun Wiesenmüller: Basiswissen RDA - der Blog rund um RDA und das Lehrbuch http:// www.basiswissen-rda.de/blog/ 